\title{
Application of digital-image-correlation techniques in analysing cracked cylindrical pipes
}

\author{
SHIH-HENG TUNG ${ }^{1, *}$ and CHUNG-HUAN SUI ${ }^{2}$ \\ ${ }^{1}$ Department of Civil and Environmental Engineering, National University of \\ Kaohsiung, Kaohsiung 811, Taiwan \\ ${ }^{2}$ Department of Civil Engineering, National Chung Hsing University, \\ Taichung 402, Taiwan \\ e-mail: shtung@nuk.edu.tw
}

MS received 26 June 2009; revised 7 June 2010; accepted 1 July 2010

\begin{abstract}
Cracks induced by external excitation on a material that has defects may generate the stress concentration phenomenon. The stress concentration behaviour causes local buckling, which will induce the damage of the members made of this material. Thus, developing techniques to monitor the strain variation of a cracked member is an important study. The traditional technique (such as strain gauge) can only measure the average strain of a region. The strain variation within this region cannot be determined. Therefore, it cannot sufficiently reflect the mechanical behaviour surrounding the crack. The Digital image correlation technique recently developed is an image identification technique to be applied for measuring the object deformation. This technique is capable of correlating the digital images of an object before and after deformation and further determining the displacement and strain field of an object based on the corresponding position on the image. In this work, this technique is applied to analyse the mechanics of a cylindrical pipe experiencing crack destruction. The fixing device is used to avoid shaking the specimen during the pressurizing process. The image capture instruments are fixed on the stable frame to measure the deformation of specimen accurately. Through the cylindrical pipe cracking test, the capacity of the digital image correlation technique for surveying the strain variation in a tiny region is validated. Then, the experimental results obtained using the digital image correlation analysis is used to demonstrate the crack development tendency in defect materials and the stress concentration zone.
\end{abstract}

Keywords. Digital image correlation; DIC; crack analysis.

\section{Introduction}

In the past, there are two methods for surveying strains developed in an object which in subject to external forces. One method is to measure the relative displacement between

${ }^{*}$ For correspondence 
two specific points on the surface of an object, and then estimate the strain between these two points. However, the global strain distribution of the object cannot be determined directly. The other method is to map meshes on the surface of an object before deformation occurs, and then survey the displacement of nodes surrounding these meshes after deformation. Further, the strain distribution of an object is derived from the displacement field. Contrarily, this technique is a complex and time-consuming process. The Developed Digital Image Correlation (DIC) technique (Chu et al 1985) is an image identification method for measuring object deformation. The digital images of an object before and after deformation that are captured using an optic instrument are subject to correlation analysis. The corresponding positions recorded on the image are obtained by calculating the correlation coefficient of images so that the displacement function and strain distribution of an object can be inferred. This technique is non-destructive for inspecting the whole displacement and strain field. Recently, Vellinga \& Onraet (2000), Vendroux \& Knauss (1998) utilized an electron microscope to capture specimen images in a tiny region and then executed DIC analysis for these images. These results indicated that the precision of DIC method is in the nanoscale range. For the application of DIC analysis in crack problem, Dost et al $(1999,2003)$ acquired images of nanoscale objects using an atomicforce microscope to detect nano-scale cracks forming on nanomaterials. Additionally, Tung et al (2008) inspected crack development in a masonry wall and examined the effect of crack formation on seismic resistance of masonry structures. The validity of applying DIC technique in crack observation for non-homogeneous materials was confirmed by their studies.

In recent years, mudflows induced by seismic excitation cause water pollution and pipeline damages. Cracks may form on a conduit to generate stress concentration thus threatening the safety and duration of the conduit. The phenomenon of stress concentration causes local buckling in the material surrounding the crack. In the past, applying numerical simulation to investigate material buckling is confined to the basic assumption that the material is elastic. (Sung et al 2002) The results thus obtained cannot sufficiently reflect the local buckling action inflicting destruction on material. Thus, developing a monitor system to inspect the mechanics behaviour of a cracked member is an essential and important study. The feasibility of applying DIC technique to monitor pipeline cracks is tested in this research by designing and applying a new test to be applied on a cracked cylindrical pipe for simulating a conduit subject to crack destruction.

\section{Basis theory of strain analysis using the digital image correlation method}

The DIC technique is based on the concept of search algorithm to execute image identification. The analysis procedure of the DIC method is shown in figure 1 . The analysis region is divided into several sub-images. The grayscale distribution of image is used to identify the relative positions of the same point on the specimen surface in two images. Correlation analyses of images are carried out to search the point that has the highest grayscale correlating with the initial position displacement vector so that the displacement field of specimen can be derived. After that the strain field can be calculated.

\subsection{Two-dimensional digital image correlation method}

Based on the discretization operation of finite element method (FEM), the undeformed image is meshed into several sub-images for image analyses. As shown in figure 2, the central point 


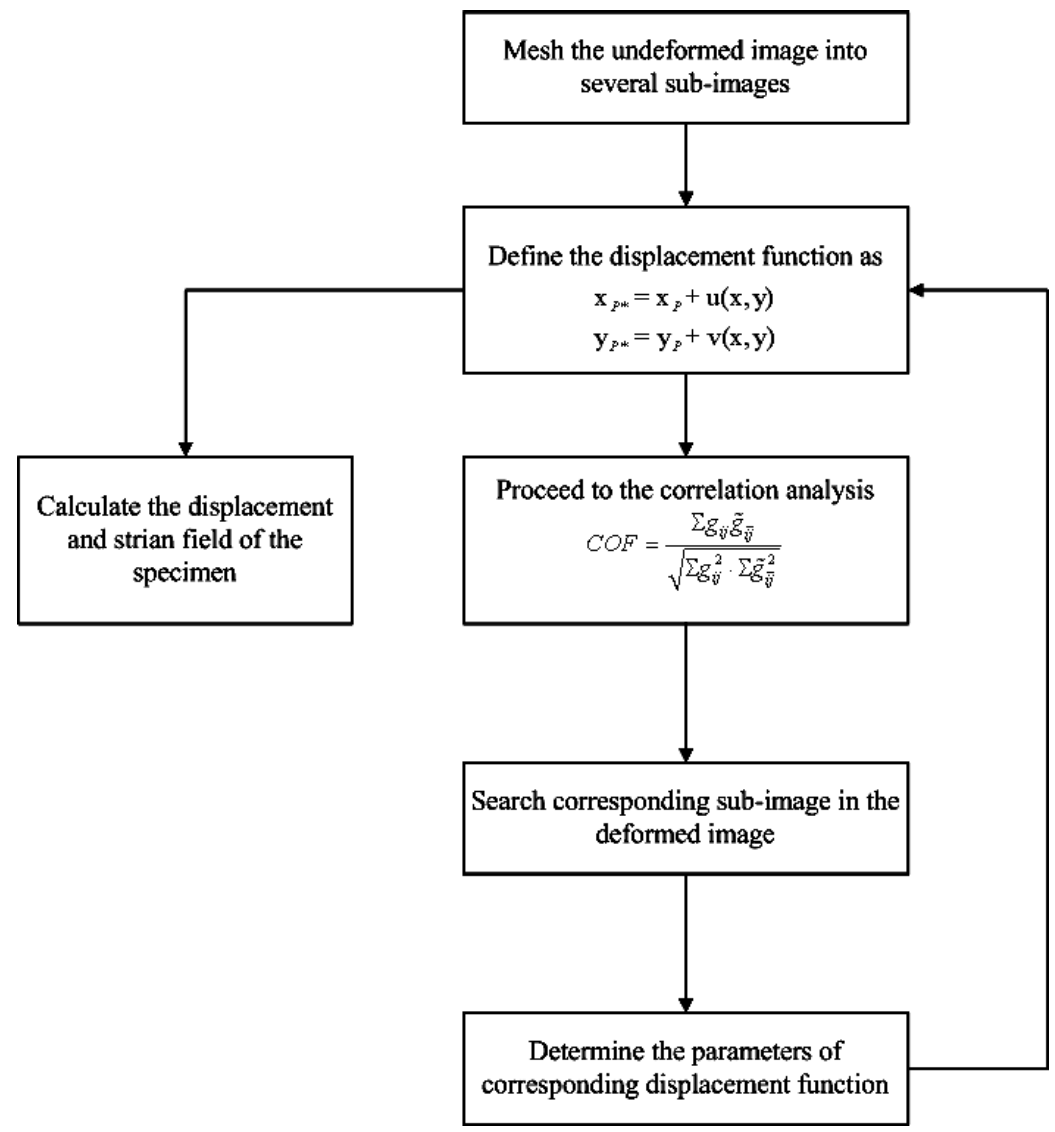

Figure 1. Analysis procedure of digital image correlation method.

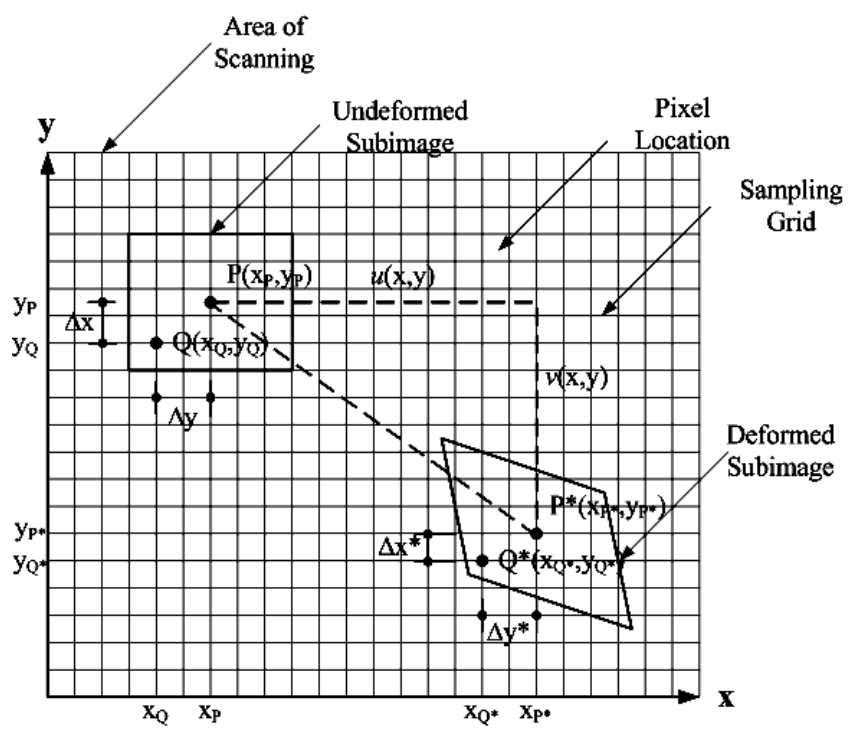

Figure 2. Corresponding relation of deformed and undeformed subimages. 

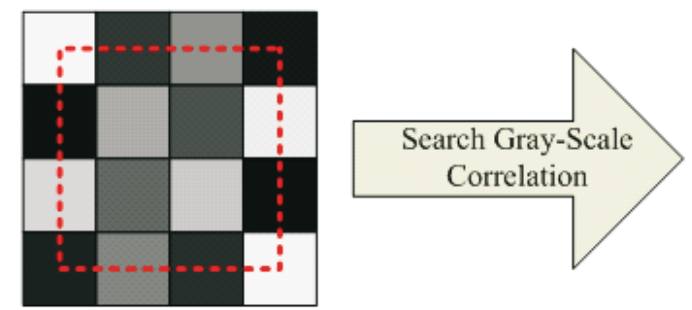

Undeformed Sub-image

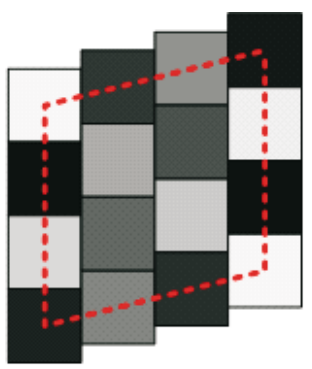

Deformed Sub-image

Figure 3. Gray-scale correlation between deformed and undeformed sub-image.

prior to deformation is point $P$; it is shifted to point $P *$ after deformation. The functional relationship is expressed as:

$$
\begin{aligned}
& x_{P *}=x_{P}+u(x, y) \\
& y_{P *}=y_{P}+v(x, y),
\end{aligned}
$$

where $\left(x_{P}, y_{P}\right)$ and $\left(x_{P *}, y_{P *}\right)$ are the coordinates of point $P$ before and after deformation. $u(x, y)$ and $v(x, y)$ are the displacement functions in $x$ - and $y$-direction seperately. Calculating the grayscale correlation coefficient will lead to finding the corresponding relationship between deformed and undeformed sub-images for establishing the deformation of sub-image as shown in figure 3 . The correlation coefficient is defined as:

$$
C O F=\frac{\Sigma g_{i j} \tilde{g}_{\overline{i j}}}{\sqrt{\Sigma g_{i j}^{2} \cdot \Sigma \tilde{g}_{\overline{i j}}^{2}}},
$$

where $g_{i j}$ and $\tilde{g}_{i \bar{j}}$ are the grayscale of the undeformed sub-image on coordinate $(i, j)$ and deformed sub-image on coordinate $(\bar{i}, \bar{j})$ respectively. When the deformed sub-image corresponds exactly to the undeformed sub-image, the correlation coefficient between both subimages equals 1 . Accordingly, the optimal parameters of equation (1) are determined based on the results of correlation analyses. Thus, the displacement and strain field can be computed individually.

\subsection{Calculation of strain field}

Green-Lagrange's tensor $E$ is defined as:

$$
E=\frac{1}{2}\left[F^{T} \otimes F-I\right]
$$

where, $F$ is gradient tensor of displacement field, and $I$ is unit matrix. Tensor $E$ is rewritten into the function of displacement field as follows (Chu et al 1985):

$$
E_{i j}=\frac{1}{2}\left(u_{i, j}+u_{j, i}\right)+\frac{1}{2} u_{k, i} u_{k, j},
$$


where, $i, j, k \in(x, y)$ and $u_{i, j}=\partial u_{i} / \partial j$. Strain is computed below:

$$
\begin{aligned}
& \varepsilon_{x x}=\frac{\partial u_{x}}{\partial x}+\frac{1}{2}\left[\left(\frac{\partial u_{x}}{\partial x}\right)^{2}+\left(\frac{\partial u_{y}}{\partial x}\right)^{2}\right] \\
& \varepsilon_{y y}=\frac{\partial u_{y}}{\partial y}+\frac{1}{2}\left[\left(\frac{\partial u_{x}}{\partial y}\right)^{2}+\left(\frac{\partial u_{y}}{\partial y}\right)^{2}\right] \\
& \varepsilon_{x y}=\frac{1}{2}\left(\frac{\partial u_{x}}{\partial y}+\frac{\partial u_{y}}{\partial x}\right)+\frac{1}{2}\left[\frac{\partial u_{x}}{\partial x} \frac{\partial u_{x}}{\partial y}+\frac{\partial u_{y}}{\partial x} \frac{\partial u_{y}}{\partial y}\right] .
\end{aligned}
$$

\subsection{Selection of sub-image size}

The displacement field shows discontinuity at both sides of a crack, and hence the correlation coefficient for the sub-images surrounding a crack is significantly reduced. The selection of sub-image size will affect the capability to resolve the strain variation. The strain close to the crack tip and specimen edge varies fast and can only be analysed with small sub-image, but once the sub-image is too small, the analysis accuracy of the displacement will be affected. Therefore, the size of the sub-image has its limit. The appropriate sub-image size must be determined in accordance with the requirement of every analysis case. The sub-image size used in this study is 32 pixels.

\section{Cylindrical pipe test}

The objective of this experiment is to demonstrate that the DIC technique is effective in probing the stress concentration behaviour of a cracked cylindrical pipe that is designed to simulate a conduit subject to longitudinal cracking. The internal pressure applied on the cylindrical pipe is to simulate the conduit conveying water. Results of numerical simulation in this experiment are used to confirm the validity of the DIC technique. Furthermore, the stress concentration zone and the crack progress tendency are also investigated through DIC analyses.

\subsection{Experimental set-up}

Figure 4 indicates the assemblage of the experimental equipment that includes the following components:

3.1a Cylindrical pipe with a longitudinal crack: The cylindrical pipe is made of AISI Type $304 \mathrm{~N}$ Stainless Steel (Modulus of Elasticity = $193 \mathrm{MPa}$, Tensile Yield Strength = $330 \mathrm{MPa}$ ). The length of cylindrical pipe is $100 \mathrm{~mm}$ and the pipe diameter is $15.6 \mathrm{~mm}$. The thickness of pipe is $0.1 \mathrm{~mm}$. The longitudinal crack is $0.38 \mathrm{~mm}$ in width and $20 \mathrm{~mm}$ in length. A rubber spacer is attached inside the cylindrical pipe using DC-795 silicone to prevent leaking. A sketch of the specimen used in this experiment is shown in figure 5.

3.1b Pressurizing system: The pressurizing system consists of a hydraulic hand pump connected to the pipe with a quick connector for pressurizing the cylindrical pipe. The pump has dimensions of $510 \mathrm{~mm} \times 140 \mathrm{~mm} \times 150 \mathrm{~mm}$, and a $900-\mathrm{cm}^{3}$ oil reservoir volume; it delivers a maximum working pressure of $700 \mathrm{~kg} / \mathrm{cm}^{2}$. 


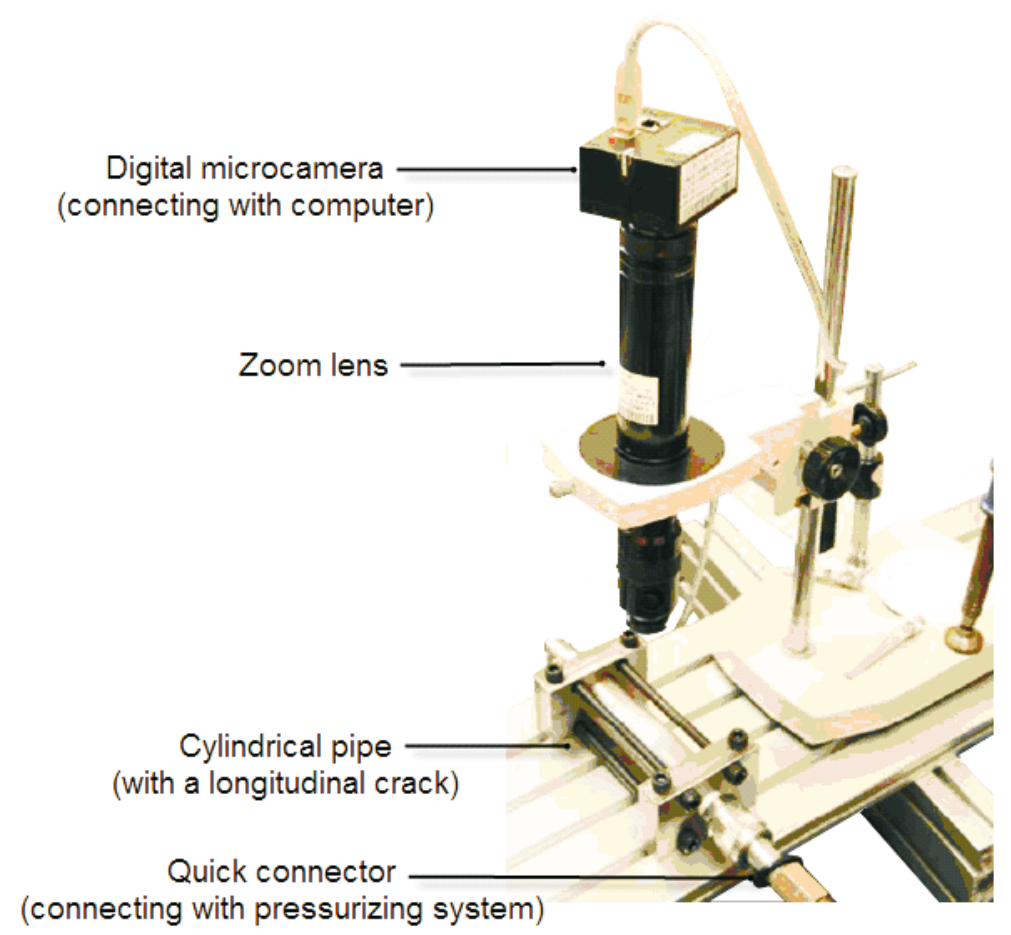

Figure 4. Assemblage of the cracked cylindrical pipe test.

3.1c Image capture instruments: The digital microcamera assemblies with the zoom lens to capture images are connected with a computer for capturing images and recording the image data. The model of the digital microcamera is NexShot2C-3.1M. It is a CMOS camera with a resolution of 3 mega pixels. The zoom lens is a Navitar Zoom 6000 series lens with a zoom range from $0.7 \mathrm{X}$ to $4.5 \mathrm{X}$. Additionally, the fibre-optic illuminator provides a high intensity illumination to improve the quality of digital images. The fixing device is used to avoid shaking the specimen during the pressurizing process.

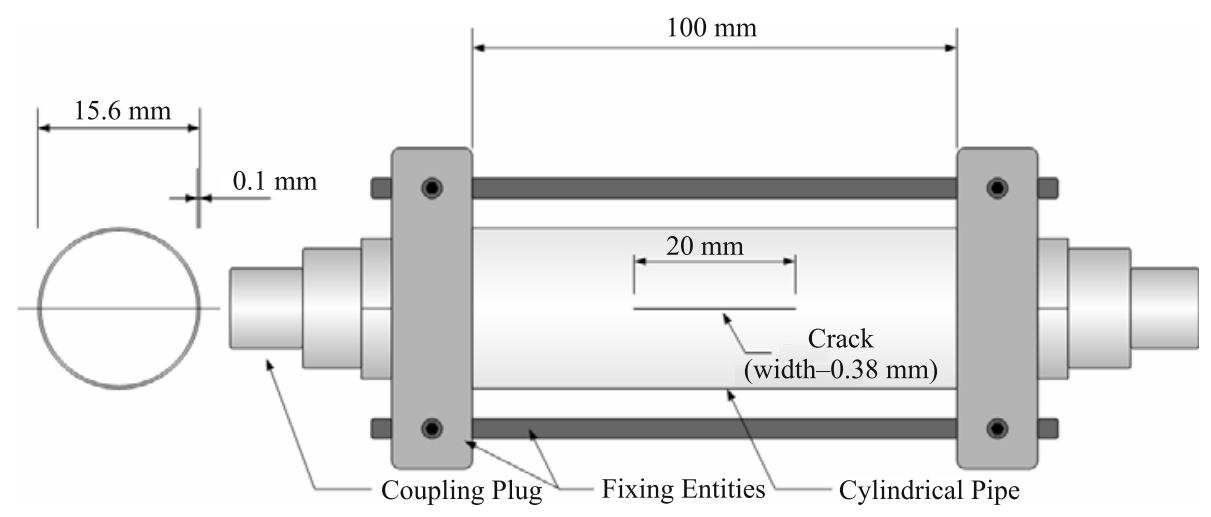

Figure 5. Equipment of the cylindrical pipe test. 


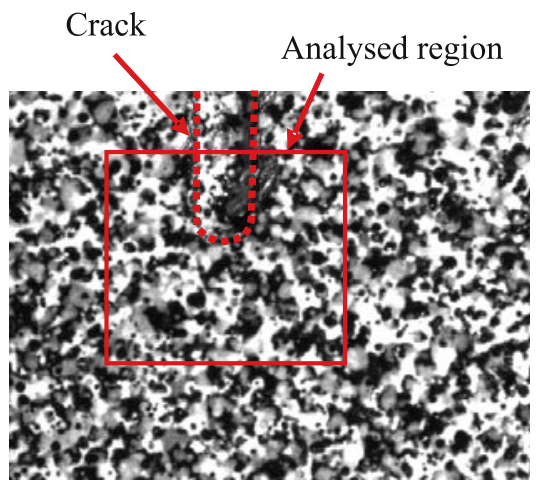

(a) 0 bar

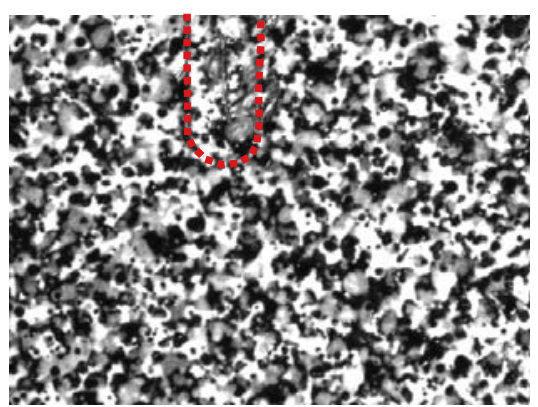

(c) $30 \mathrm{bar}$

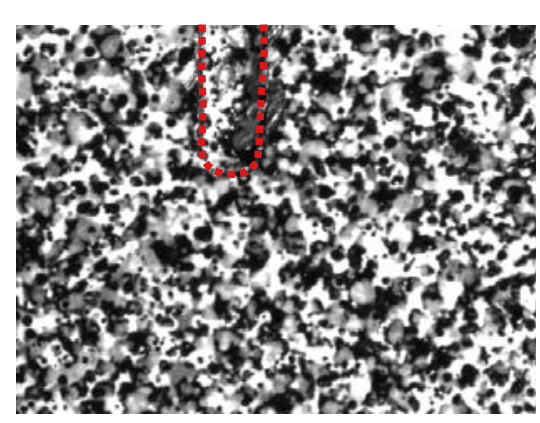

(b) 10 bar

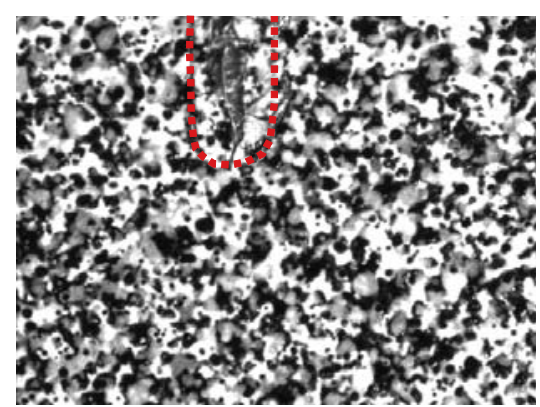

(d) $50 \mathrm{bar}$

Figure 6. Specimen surfaces under different internal pressure.

As shown in figure 6, the black and white 'structural speckle' is sprayed on the surface of the cylindrical pipe to make the non-uniform distribution of grayscale on images. This grayscale distribution offers an important character at recognizing the corresponding subimages between undeformed and deformed images. The image is recorded at an increment of $5 \mathrm{~kg} / \mathrm{cm}^{2}$ internal pressure applied to the cylindrical pipe.

\subsection{Test results}

Figure 6 shows the increment of the crack width causing the rubber spacer to be squeezed when the internal pressure increases from 0 to $50 \mathrm{~kg} / \mathrm{cm}^{2}$. There is no visual destruction of the cylindrical pipe for the internal pressure less than $25 \mathrm{~kg} / \mathrm{cm}^{2}$. The crack is filled with the silicone as seen on the digital image. As the internal pressure reaches to $50 \mathrm{~kg} / \mathrm{cm}^{2}$, the width of the crack increases on the digital image. Then, the rubber spacer is gradually squeezed from the crack during pressurizing process. At $65 \mathrm{~kg} / \mathrm{cm}^{2}$, the crack progress is visualized and the internal pressure in the cylindrical pipe is suddenly reduced due to the leak of the hydraulic fluid because the rubber spacer is peeling off caused by the destruction of the silicone.

\section{Image analysis}

Image analyses are carried out in a rectangular region (width: $1.465 \mathrm{~mm}$ and length: $1.647 \mathrm{~mm}$ ) which the crack tip centers on. The actual dimension of the image pixel is $0.003125 \mathrm{~mm}$. 


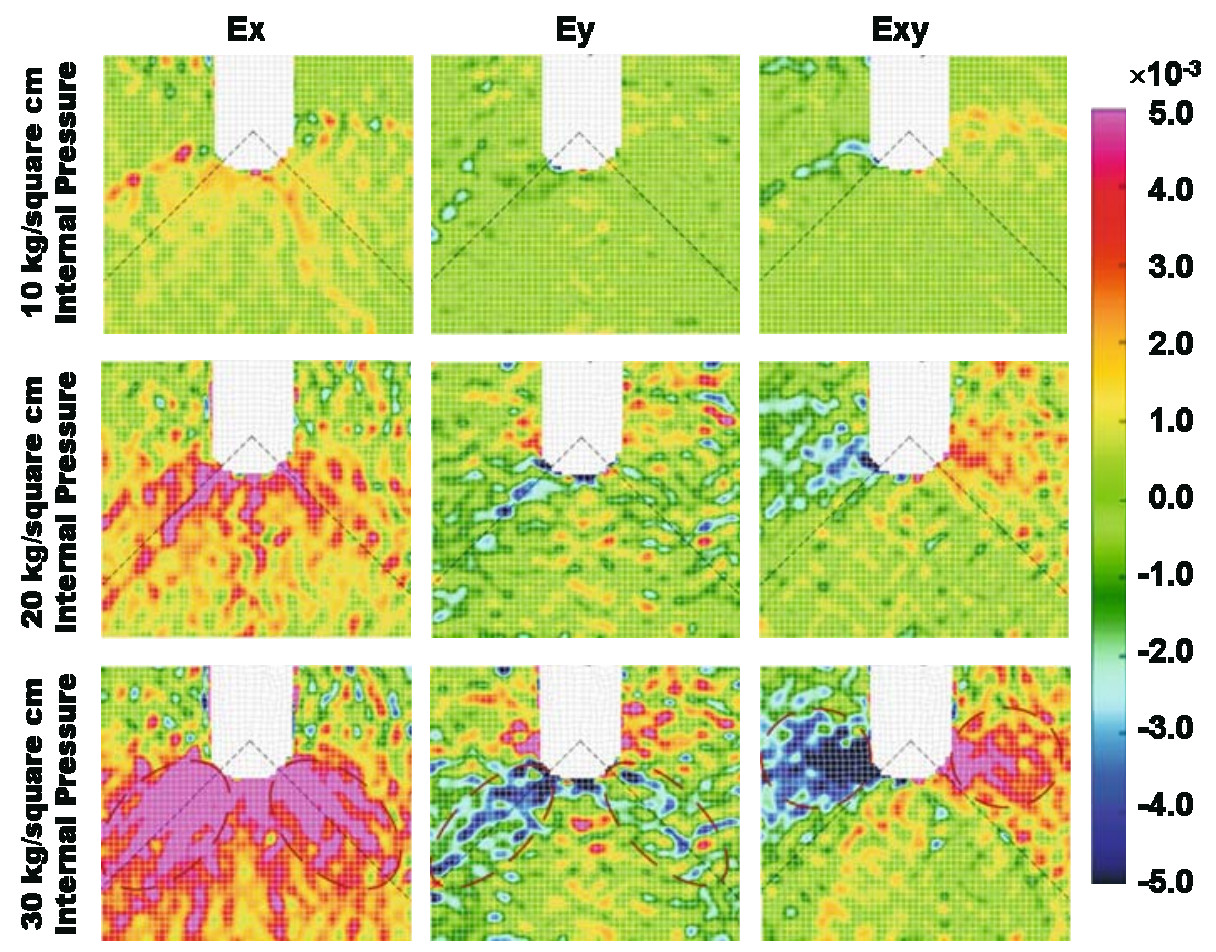

Figure 7. Distribution of strain fields in different loading phases by DIC analysis.

For meshing the un-deformed image into several sub-images, the designation of sub-image size affects the resolution of strain analyses. Larger sub-images offer higher accuracy of displacement field analyses but fail to expose local strain variations. The size of sub-image is fixed at 32 pixels to yield an accurate description of strain variations in the surrounding of the crack. The positioning accuracy of the DIC method is about 0.01 pixels in the laboratory. For a 32 pixels sub-image, the corresponding strain accuracy is about $2 \times 10^{-4}$.

As shown in figure 7, the distribution of strain fields in different loading phases is obtained from DIC analyses. Under the exertion of lower internal pressure, the magnification of horizontal strain is evident at the crack tip; the vertical strain and the shear strain in the analysis scope exhibit almost uniform distribution. With the increment of internal pressure, the existence of the crack induces the non-uniform deformation in the surrounding of the crack. The non-uniform distribution of strain field may be approximately divided into three regions. Due to the exertion of internal pressure, the relative movement of both sides of the crack causes significant increment of the shear strain in the regions beside the crack. Two regions beside the crack rotate around the crack tip in opposite directions and exert compression to the region in front of the crack tip. This leads to compressive stress concentration to generate local buckling behaviour for steel material. Because of the interaction of forced regions, the horizontal and vertical strains in \pm 45 degree directions are abruptly magnified to make material yielding. Subsequently, the magnification of shear strain follows the direction that is perpendicular to the longitudinal axis from the crack tip. This phenomenon involves the strain singularity induced by local buckling. In different loading phases, the crack development tendency can be unequivocally indicated from the variation gradient of the strain field. With adding internal 


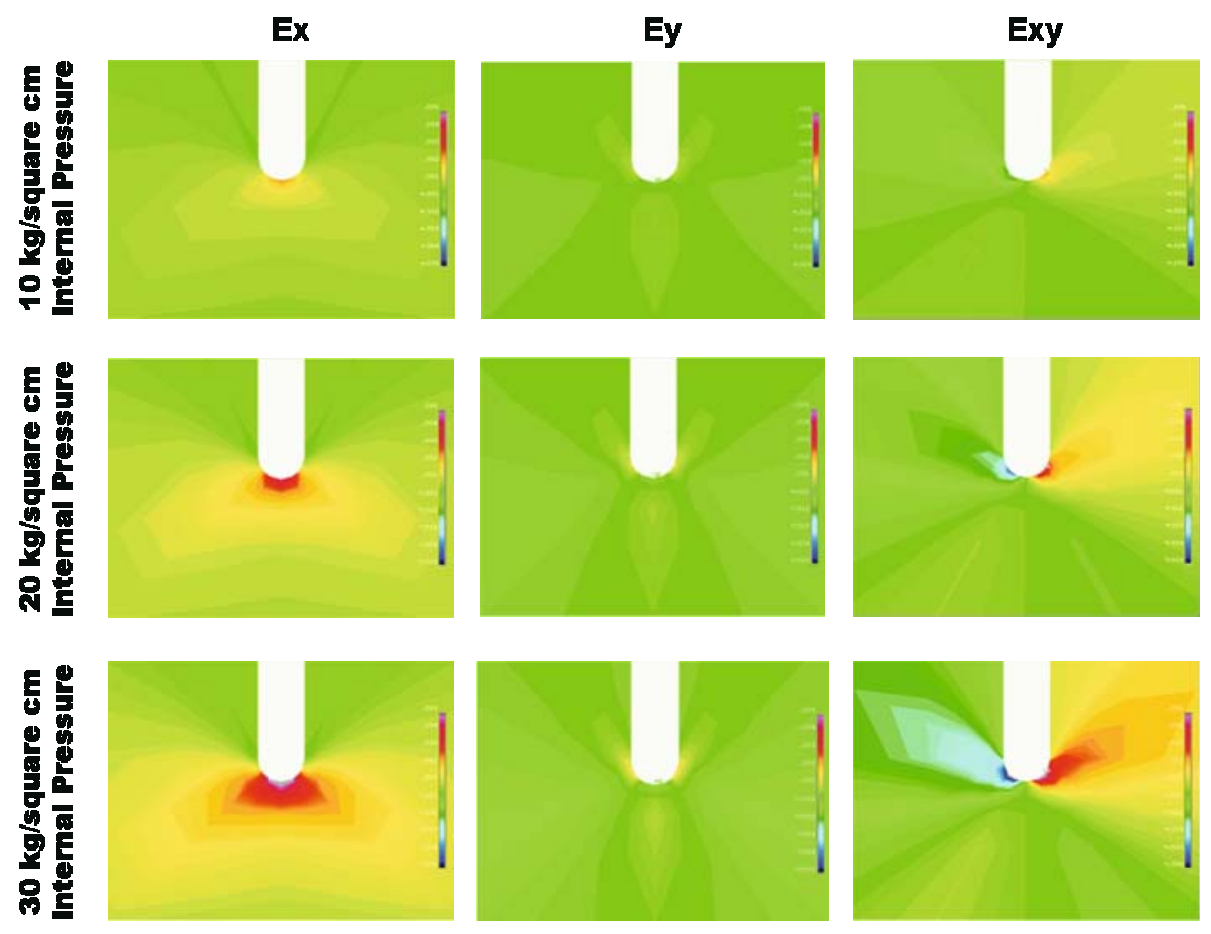

Figure 8. Distribution of strain fields in different loading phases by numerical analysis.

pressure to the specimen, the stress concentration zone follows the \pm 45 degree direction to develop forward and further expands to the region in front of the crack tip.

In order to examine the accuracy of image analysis results, numerical simulation of this experiment is carried out for verifying the laboratory observations. Mathematical modelling of the cracked cylindrical pipe is established using the infinite-small element method, which is based on the assumption of linear elastic material (Go \& Lin 1990). For the infinite-small element method, the analysis model is meshed into many similar elements at the crack tip. The quantity of elements is determined by the demand of the precision. Based on the mechanical characteristic of similar element possessing similar stiffness matrix, the stress singularity at the crack tip can be reflected by this method. The analysis program in this paper is established by Sung et al (2002). Figure 8 shows the results of numerical analyses on the distribution of strain field under different loading pressures. The strain distribution based on numerical analyses shows that the development of the horizontal strain traces the \pm 45 degree direction to expand forward. Then, the shear strain is magnified from the crack tip following the peripheral direction. These phenomena match the results of image analysis. The observation also proves the accuracy of the qualitative analyses by using the DIC technique. However, figures 7 and 8 show that the strain magnitude obtained using numerical analyses is lower than that obtained using image analyses. Thus, extracting the horizontal strains in the longitudinal and the 45 degree directions for comparison between both analyses are shown in figure 9 with the transverse axis indicating the distance from the crack tip. In the longitudinal direction, the horizontal strain of image analyses is quite closed to that of numerical analyses under $10 \mathrm{~kg} / \mathrm{cm}^{2}$ internal pressure. The comparison proves the accuracy of quantitative analyses 

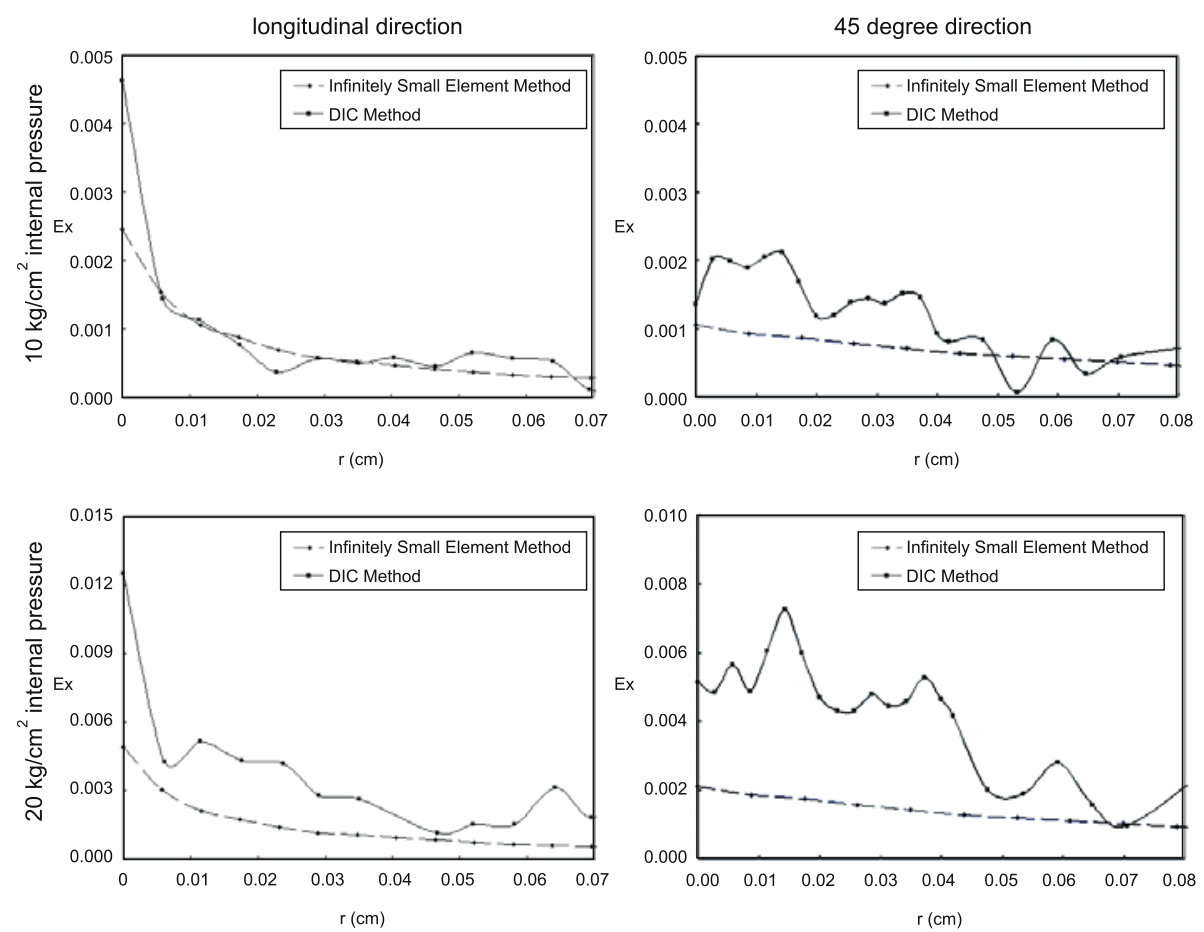

Figure 9. Comparison between image analysis and numerical analysis for the horizontal strain in the longitudinal and 45 degree direction.

using the DIC technique for linear elastic materials. When the horizontal strain exceeds the yield strain of material, the schema of image analyses deviates from that of numerical analyses as shown in figure 9. The reason of this deviation is that the infinite-small element method, which is based on the assumption about linear elastic material, underestimates the horizontal strain at the material yielding (yield strain of stainless steel $=0.0017$ ). The same reason accounts for the deviation near the crack tip that significantly increases due to the stress concentration causing the material yielding. For the horizontal strain in the 45-degree direction, the DIC analysis clearly presents the magnification of horizontal strain caused by local buckling mechanism. Thus, the quantities of horizontal strain acquired through image analyses are larger than those acquired using numerical simulation. Additionally, the incapability of numerical analysis to expose the phenomenon of local buckling behaviour induced by stress concentration is also demonstrated.

\section{Conclusion}

This research work demonstrates that the DIC method is an appropriate technique for image identification with high precision. This technique is applied to analyse the mechanics of a fixed cylindrical pipe experiencing crack destruction. In order to assuring the accuracy of DIC method, the specimen and image capture instruments must be fixed on the stable device to avoid shaking effect. According to analysis results presented in this paper, the strain distribution of DIC analysis is similar to that of numerical simulation under the exertion of lower 
internal pressure. Due to the assumption of linear elastic material for numerical simulation, the incapability of numerical analysis to reflect local mechanical behaviour is also indicated at the specimen subjected to higher internal pressure. Results of laboratory studies confirm that the DIC technique is reliable in investigating a crack problem, and presenting the phenomenon of stress concentration that causes strain singularity and local buckling behaviour. This technique improves the weak points and restricts numerical simulation for analysing a crack problem. The feasibility of DIC method for surveying the strain distribution that causes local buckling, warping or crumpling behaviour on materials is also validated. Thus, the DIC method is used to monitor pipelines, bridges, buildings, etc. and to identify the stress concentration zone in the defect materials.

We thank the National Science Council of Taiwan for supporting this research through grant No. NSC 97-2625-M-390-001 and NSC 97-3114-E-167-001.

\section{References}

Chu T C, Ranson W F, Sutton M A, Peters W H 1985 Application of digital-image-correlation techniques to experimental mechanics. Experimental Mech. 25: 232-244

Dost M, Rümmler N, Kieselstein E, Erb R, Hillmann V, Großer V 1999 Correlation analysis at grey scale patterns in an in-situ measuring module for microsystem technology. Materials mechanics Fracture mechanics - micromechanics, (eds.) T Winkler, A Schubert, Berlin/Chemnitz, 259-266

Dost M, Vogel D, Winkler T, Vogel J, Erb R, Kieselstein E 2003 How to detect Edgar Allan Poe's 'purloined letter' - or: Cross correlation algorithms in digitised video images for object identification, movement evaluation and deformation analysis. Proceedings of SPIE Vol. 5048, Nondestructive Detection and Measurement for Homeland Security (USA: Bellingham, WA).

Go C G, Lin Y S 1990 Infinitely small element for the problem of stress singularity. Computer and Struct. 37(4): 547-551

Sung W P, Shih M H, Go C G 2002 Analysis Modelling for the Sequence of Local Buckling of Crack Thin Plate under Tension. J. Zhejiang Univ. SCIENCE A. 8(9): 1371-1379

Tung S H, Shih M H, Sung W P 2008 Development of digital image correlation method to analyse crack variations of masonry wall. Sādhanā (Academy Proceedings in Engineering Sciences) 33(6): $767-779$

Vellinga W P, Onraet S 2000 Measurement of Strain Fields in the Micron Range. Proceedings of the joint Meeting of the BVM and the NVvM 2000 in Papendal, Arnhem 110-111

Vendroux G, Knauss W G 1998 Submicron Deformation field measurements: Part 2. Improved digital image correlation. Experimental Mech. 38(2): 86-92 